\title{
¿Qué ocurre a los 24 meses del inicio del tratamiento en una muestra de personas con dependencia de la cocaína?
}

\author{
Ana lópez*; Elisardo Becoña*; Isabel Vieitez**; Jesús Cancelo**; Juan Sobradelo**; \\ José $M^{a}$ García***; Avelina Senra***; Lorena Casete****; Ma Teresa Lage**** \\ * Departamento de Psicología Clínica y Psicobiología. Facultad de Psicología. Universidad de Santiago de Compostela. \\ * Psicólogos Clínicos. Unidad Asistencial Alborada. Vigo (Pontevedra). \\ ** Psicólogos Clínicos. Unidad Asistencial del Ayuntamiento de Pontevedra. \\ ****Psicólogos Clínicos. Unidad Asistencial ACLAD. A Coruña. \\ Enviar correspondencia a: \\ Ana López Durán. Universidad de Santiago de Compostela. Departamento de Psicología Clínica y Psicobiología. \\ Campus Universitario Sur. 15782 Santiago de Compostela. E-mail: ana.lopez@usc.es
}

\section{RESUMEN}

La evolución de las personas que están en tratamiento por consumo de cocaína en Centros de Drogodependencias públicos, es un tema relevante debido al notable incremento de la demanda de estos tratamientos en los últimos años. El objetivo del presente estudio es analizar la evolución de personas con dependencia de la cocaína que han recibido tratamiento en Centros públicos de Drogodependencias de Galicia, tras dos años de haberse realizado una evaluación inicial. La muestra el presente estudio es de 38 sujetos que formaban parte de una muestra inicial más amplia ( $N=$ 115), y las variables que se analizan son: el consumo de sustancias, el ajuste social con la escala del O.T.I., problemas con la justicia y el malestar psicológico percibido por el sujeto evaluado con las subescalas e índices del SCL-90 R. Los resultados obtenidos son: a los dos años, el 78.9\% de la muestra localizada no ha consumido cocaína en el mes previo a la evaluación, lo cual representa un $26 \%$ de la muestra inicial, y una disminución del malestar percibido por el sujeto. Estos resultados indican una evolución positiva de la muestra de personas en tratamiento por dependencia de la cocaína a los dos años que ha sido analizada en el presente estudio.

Palabras clave: cocaína, seguimiento, tratamiento, evaluación, abstinencia

\section{ABSTRACT}

The evolution of people in treatment for cocaine dependence at public Drug Abuse Centers is a relevant topic in view of the notable increase in demand for such treatment in recent years. The aim of the present study was to analyze the evolution of people with cocaine dependence receiving treatment at public Drug Abuse Centers in Galicia (NW Spain), two years after the initial assessment for inclusion on the treatment program. The sample for the present study was 38 participants who formed part of a larger initial sample $(N=115)$. The variables analyzed were: substance use, social adjustment on the O.T.I. scale, problems with the legal system and perceived psychological distress as assessed with the scales and indices of the SCL-90 R. The results indicate that, after two years of treatment, $78.9 \%$ of those in the sample located ( $26 \%$ of the initial sample) had not consumed cocaine in the month prior to assessment, and that there was a reduction in psychological distress. These results indicate a positive evolution after two years of the sample of people in treatment for cocaine dependence assessed in this study.

Key words: cocaine, follow-up, treatment, assessment, abstinence

\section{INTRODUCCIÓN}

El consumo de cocaína tiene unos niveles muy altos en España. Los datos de la última encuesta domiciliaria realizada por el Plan Nacional sobre Drogas (PNSD, 2007) apuntan que el $7.0 \%$ de las personas entre 15 y 64 años la han probado alguna vez, el 3.0\% consumió cocaína durante el último año y un $1.6 \%$ lo hizo el último mes. El grupo de edad de 15 a
34 años es el que tiene un porcentaje de consumo de cocaína más alto en el último año (5.2\%). En los últimos 10 años el incremento ha sido notable, así la proporción de consumidores en los últimos 12 meses pasó de $1.8 \%$ en 1995 a un $3.0 \%$ en 2005 .

Este incremento en el consumo, también ha provocado un incremento en la demanda de tratamientos en los Centros de Drogodependencias. Como es un fenómeno reciente, en nuestro país son escasos los 
estudios de seguimiento de la evolución de las personas que están en tratamiento por problemas con la cocaína (López, Becoña, García, Senra, Cancelo, Estévez et al., 2006; López, Becoña, Lloves, Moneo, Vieitez, Cancelo et al., 2007; Pedrero, Puerta, Segura y Martínez, 2004; Sánchez-Hervás, Morales, Tomás, Molina, del Olmo, Bosch et al., 2002).

En la realización de estudios de seguimiento hay diversas dificultades. Entre ellas destacan la pérdida de sujetos que forman la muestra inicial y cómo evaluar la abstinencia de cocaína. El número de sujetos que forman la muestra inicial va disminuyendo a media que avanza el tratamiento, principalmente por el abandono del tratamiento por parte de los sujetos o las dificultades de localización tras el alta terapéutica. En el estudio de Carroll, Power, Bryant y Rounsaville (1993) tras un año de tratamiento sólo pudieron evaluar al 32\% de la muestra inicial. Carroll, Nich, Ball, McCance, Frankforter y Rousaville (2000) evaluaron tras un año al 37.5\% de los sujetos de la muestra inicial. Respecto a la evaluación de la abstinencia en el consumo de cocaína, hay diversos criterios para analizarla: la realización de consumos durante un periodo previo a la realización de la evaluación (Crist-Christoph, Siqueland, Blaine, Frank, Luborsky, Onken et al., 1999; McMahon, Kouzekanani y Malow, 1999) o la abstinencia total en el consumo durante el periodo de tiempo que dura el seguimiento (Bernstein, Bernstein, Tassiopoulos, Heeren, Levenson y Hingson, 2005). Reiber, Ramírez, Parent y Rawson (2002) defienden el uso de la variable consumo de sustancias en el último mes, ya que predice la ocurrencia de posteriores nuevos consumos.

El tratamiento que reciben las personas con dependencia de la cocaína que acuden a Centros de Drogodependencias en nuestro entorno es, en la mayoría de los casos, un tratamiento consistente en una intervención psicológica de tipo cognitivo-conductual con prevención de la recaída que es el tratamiento que tiene evidencia científica de ser eficaz (Crist-Christoph et al., 1999; Secades-Villa, García-Rodríguez, Álvarez, Río, Fernández-Hermida y Carballo, 2007). En estos momentos, no existe un tratamiento farmacológico que haya demostrado ser eficaz para el tratamiento de la dependencia de la cocaína. El planteamiento actual es acompañar, en los casos en los que es necesario por la psicopatología que presenta, el tratamiento psicológico con tratamiento farmacológico (Solé, 2001).

El objetivo del presente estudio es analizar la evolución de personas con dependencia de la cocaína que han recibido tratamiento en Centros de Drogodependencias de Galicia en las áreas de consumo de sustancias, funcionamiento social, problemas con la justicia y malestar psicológico percibido por el sujeto, dos años después del inicio del tratamiento.

\section{MÉTODO}

\section{Participantes}

La muestra del presente estudio está formada por 38 sujetos, 35 hombres y 3 mujeres, con una edad media de 31 años, evaluada dos años después de su demanda de tratamiento. Estos sujetos formaban parte de una muestra inicial más amplia, 115 personas con dependencia de la cocaína que comenzaron el tratamiento por problemas con el consumo de esta sustancia en distintos Centros de Drogodependencias de Galicia: U.A.D. Pontevedra, A.C.L.A.D. A Coruña, y Alborada Vigo (López et al., 2006). Por lo tanto, hemos evaluado a un 33.0\% de la muestra inicial después de 24 meses, ya que éstos han sido los localizados a los dos años.

A los dos años de realizarse la evaluación inicial, se vuelve a contactar con los 115 sujetos. Únicamente fueron localizados 38. Tres personas habían fallecido (un suicidio y dos fallecimientos por enfermedad), y el resto no fueron localizados por cambio en el lugar de residencia o en el número de teléfono, o bien no acudieron a la cita para realizar la evaluación. De las 38 personas que fueron evaluadas, 22 continuaban en tratamiento, 12 habían recibido alta terapéutica, 2 habían abandonado el tratamiento, una persona estaba derivada en otro centro y una persona estaba en la cárcel.

Los criterios de inclusión para formar parte del estudio inicial eran: estar en tratamiento por consumo de cocaína como droga principal, existencia de dependencia de la cocaína según los criterios del DSM-IV-TR, y llevar un mínimo de tres semanas y un máximo de seis semanas abstinente en el consumo de cocaína. Los criterios de exclusión fueron: presencia de alteraciones psicóticas graves e incapacidad de responder a los instrumentos de evaluación utilizados (López et al., 2006; López et al., 2007). Ninguno de los participantes en el estudio se encontraba en un programa de mantenimiento con metadona.

\section{Instrumentos de evaluación}

Antes del tratamiento todos los sujetos cumplimentan los siguientes cuestionarios, como se puede ver en López et al. (2006): Entrevista Clínica Estructurada para el DSM-IV (SCID) para evaluar la dependencia de la cocaína (First, Spitzer, Gibbson, Williams y Smith-Benjamin, 1998), Test para la Identificación de Trastornos por Uso de Alcohol (AUDIT; Saunders, Aasland, Babor, De la Fuente y Grant, 1993), Inventario de Depresión de Beck (BDI; Beck Rush, Shaw y Emery, 1979), Inventario de Ansiedad Estado-Rasgo (STAI; Spielberger, Gorsuch y Luchene, 1971), Inventario Clínico Multiaxial de Millon (MCMI-II; Millon, 1999), 
y Cuestionario de 90 Síntomas Revisado (SCL-90-R; Derogatis, 2002).

Transcurridos 24 meses de la primera evaluación, los sujetos cumplimentaron los siguientes cuestionarios:

Cuestionario de 90 Síntomas Revisado (SCL-90-R) para evaluar el malestar subjetivo y determinados síntomas, en su versión española (Derogatis, 2002). Utilizamos las puntuaciones de los tres índices generales: Índice General Sintomático (IGS), Total de Síntomas Positivos (TSP) e Índice de Malestar (IM). Este cuestionario se había administrado también en la evaluación inicial, hace dos años.

Escala de funcionamiento social del O.T.I. (Indicador del tratamiento de la adicción a opiáceos), en su versión española (González-Saiz, Salvador, Martínez, López, Ruz y Guerra, 1997). Esta escala ha sido diseñada para evaluar el funcionamiento social en personas con problemas con el consumo de opiáceos, concretamente analiza la integración social (empleo, estabilidad social y conflictos interpersonales), el apoyo social, y la implicación en ambientes relacionados con el consumo de drogas. Las puntuaciones se agrupan en gravedad baja, baja-media, media, mediaalta y alta. En el presente estudio modificamos el enunciado del ítem 11 para aplicar la escala a consumidores de cocaína, donde dice "heroína" lo sustituimos por "cocaína".

Consumo de sustancias en el mes previo a la evaluación: se evalúa la existencia de consumos de tabaco, alcohol, cannabis, cocaína, benzodiacepinas y heroína en el mes previo a la evaluación. Se analiza el número de veces y la cantidad de sustancia que han consumido el último mes.

Evaluación del área judicial. La persona debe de indicar si en la actualidad no tiene problemas judiciales, si está acusado de cometer delitos leves, si está acusado de cometer delitos importantes, si se encuentra en libertad condicional o si en la actualidad está cumpliendo condena.

Instrumento diseñado para el estudio para la Evaluación global del paciente a realizar por el terapeuta de referencia a 24 meses de la evaluación inicial (anexo 1). Cuando se evalúa a un sujeto, su terapeuta debe determinar en una escala tipo Likert de cero a cuatro cómo considera que está el sujeto en las siguientes áreas: psicopatológica, familiar/social, laboral y judicial. En el área de psicopatología, cero indica ausencia de síntomas, uno indica presencia de algunos síntomas leves, dos indica presencia de síntomas moderados, tres indica presencia de un trastorno psicopatológico grave y cuatro indica que el paciente presenta una alteración de la realidad o de la comunicación. En el área familiar/social, cero indica que hay un funcionamiento normalizado, uno que presenta algunas dificultades, dos que tiene dificultades moderadas, tres indica que tiene dificultades importantes en las relaciones y cuatro indica que hay una alteración grave en el área de las relaciones. En el área laboral, cero indica que hay un funcionamiento normalizado, uno indica que hay algunas dificultades, dos indica que hay dificultades moderadas, tres indica que hay dificultades importantes y cuatro indica que hay una alteración grave en este área. En el área judicial, cero indica que no tiene problemas judiciales, uno indica que está acusado de cometer delitos leves, dos indica que está acusado de cometer delitos importantes, tres indica que se encuentra en libertad condicional y cuatro indica que en la actualidad está cumpliendo condena. Este cuestionario ya se había administrado en la evaluación inicial, hace dos años.

\section{Procedimiento}

Dos años después de la evaluación inicial, los psicólogos de referencia se pusieron nuevamente en contacto con los sujetos que habían sido evaluados, les administraban los cuestionarios de evaluación (Escala de Funcionamiento Social del O.T.I., SCL-90-R y una sencilla escala de evaluación del área judicial), y evaluaban el consumo de sustancias en el último mes. En algunos de los sujetos la evaluación se hizo por teléfono o a través del correo ordinario, por no encontrarse cerca del Centro de Drogodependencias.

El terapeuta de referencia cubría la Escala de evaluación global del paciente. Todos los sujetos firmaron el consentimiento informado.

El tratamiento psicológico aplicado a las personas de esta muestra es el habitual en estos Centros de Tratamiento. La mayor parte de ellos realizan un tratamiento consistente en una intervención de tipo cognitivo-conductual con prevención de la recaída que es el que tiene evidencia científica de ser eficaz (CristChristoph et al., 1999). Si tienen otros problemas psicopatológicos se aplica psicoterapia o la medicación indicada en cada caso.

\section{Analíticas de orina}

Para determinar la existencia de consumos de cocaína, en los casos en los que fue posible, se realizaron analíticas de orina. El instrumento para realizar las analíticas de orina en todos los centros que participaron en el estudio es el ETS PLUS SYVA, que realiza análisis de tipo enzimoimnulógico.

El $47.3 \%$ de los sujetos que fueron localizados (n = 18), realizaron analíticas de orina para confirmar la abstinencia a cocaína. El resto de los participantes en el estudio $(n=20)$ no pudieron realizarla porque no podían acudir al Centro de Drogodependencias en el 
momento de la evaluación: 10 sujetos tenían alta terapéutica, 2 habían abandonado el tratamiento, 1 sujeto había sido derivado a otro centro de tratamiento y otro estaba en la cárcel. Los 6 sujetos restantes continuaban en tratamiento pero no habían realizado recientemente analíticas de orina.

\section{Tratamiento estadístico}

Se utilizó el paquete estadístico SPSS para Windows (versión 13.0). Se realizaron análisis de frecuencias y puntuaciones medias.

\section{RESULTADOS}

En primer lugar analizamos los consumos de distintas sustancias en el último mes. Respecto al consumo de cocaína, el $78.9 \%$ de la muestra $(n=30)$ no ha consumido en el mes previo a la evaluación. Por lo tanto, sólo ocho personas habían consumido cocaína en el último mes: tres personas habían consumido sólo un día, dos personas consumieron dos días, una persona consumió tres días, una persona consumió cinco días y una persona consumió diariamente cocaína en el último mes. El número de sujetos abstinentes a los 24 meses representa un $26.0 \%$ del total de la muestra inicial $(\mathrm{N}=115)$.

En la tabla 1, podemos ver los porcentajes de sujetos abstinentes a los seis, doce y veinticuatro meses. En el caso de los seis y doce meses, la abstinencia que se evalúa es continua. Es decir, todo sujeto que haya realizado como mínimo un consumo de cocaína (entre la evaluación inicial y los seis meses posteriores, y entre la evaluación inicial y los doce meses posteriores) se clasificaría como sujeto no abstinente. A los seis meses, el 30.4\% del total de la muestra estaba abstinente, y a los doce meses lo estaba el 17.4\%.

En cuanto al consumo de otras sustancias los datos son los siguientes: el $10.5 \%$ de la muestra $(n=4)$ no ha fumado tabaco en el último mes, el 31.6\% ( $n=12)$ ha fumado menos de una cajetilla al día, y el $57.9 \%$ ( $n=22$ ) ha fumado una cajetilla o más diariamente durante el último mes. Respecto al consumo de alcohol, el $26.3 \%$ ( $n=10$ ) no ha consumido alcohol en el último mes, el 55.3\% ( $n=21)$ ha consumido alcohol como máximo ocho días en el último mes, y un $18.4 \%$ ( $n=7$ ) ha bebido alcohol más de ocho días en el último mes. En el consumo de cannabis, el $63.2 \%$ de la muestra ( $n=24)$ no ha consumido cannabis en el último mes, el $13.2 \%(n=5)$ lo ha consumido como máximo ocho días, y el $23.7 \%(n=9)$ ha consumido cannabis más de 20 días en el último mes. Respecto al consumo de heroína, sólo dos sujetos (5.3\%) la consumieron en el último mes.
Tabla 1. Porcentaje de abstinencia en el consumo de cocaína a los 6, 12 y 24 meses.

\begin{tabular}{|cc|}
\hline A los 6 meses(1) & $\begin{array}{c}\% \text { de sujetos } \\
\text { abstinentes en el } \\
\text { consumo de cocaína }\end{array}$ \\
$\%$ del total ( $N=115)$ & 30.4 \\
$\%$ de los localizados $(n=92)$ & 38.0 \\
A los 12 meses(1) & 17.4 \\
$\%$ del total ( $N=115)$ & 21.5 \\
$\%$ de los localizados $(n=93)$ & \\
A los 24 meses(2) & \\
$\%$ del total ( $N=115)$ & \\
\end{tabular}

(1) Abstinencia continuada; (2) Abstinencia en el último mes

En cuanto a la escala de funcionamiento social del O.T.I., el $71.1 \%$ de la muestra $(n=27)$ tiene un funcionamiento social adecuado (gravedad baja), el 15.8\% tiene una gravedad baja-media $(n=6)$, el 10.5\% tiene una gravedad media $(n=4)$, y sólo un sujeto $(2.6 \%)$ tiene una gravedad media-alta en esta área.

Respecto a la evaluación en el área judicial, el 73\% de la muestra no tiene problemas con la justicia ( $n=$ 27), seis sujetos (16.2\%) están acusados de cometer delitos leves, tres están acusados de cometer delitos importantes (8.1\%), y un sujeto se encuentra cumpliendo condena en el momento de la evaluación (2.7\%). Un sujeto no respondió en esta escala.

En el SCL-90-R las puntuaciones medias obtenidas en las subescalas son las siguientes: en la escala de somatización la puntuación media es de 0.73 (D.T. = $0.67)$, en la escala de obsesión compulsión la media es de 0.79 (D.T. = 0.65), en la escala de sensibilidad interpersonal la media es $0.67(\mathrm{D} . \mathrm{T}=0.71)$, en la escala de depresión la media es de 0.81 (D.T. = 0.79), en la de ansiedad la media es de 0.59 (D.T. $=0.62)$, en la de hostilidad la media es 0.40 (D.T. $=0.43$ ), en la de ansiedad fóbica la media es de 0.33 (D.T. $=0.42$ ), en la de ideación paranoide la media es de 1.23 (D.T. = 2.72), y en la de psicoticismo la media es de 0.47 (D.T. $=0.51$ ). En los índices generales las puntuaciones medias son las siguientes: en el índice general sintomático la puntuación media es de 0.66 (D.T. = 0.55), en el total de síntomas positivos la media es de 34.55 (D.T. = 19.32), y en el índice de malestar la media es 
de 1.58 (D.T. = 0.45). Todas las puntuaciones medias son inferiores a los dos años de la evaluación inicial, excepto en las subescalas de somatización e ideación paranoide. Como se puede ver en la figura 1, a los dos años de realizada la evaluación inicial las puntuaciones medias en las subescalas y en los índices, disminuyen acercándose más a las puntuaciones utilizadas como baremos de la población normal.

También hay que destacar que a los dos años, el porcentaje de personas que se sitúan en las tablas de población normal del SCL-90-R es superior (42.1\%, $n=16$ ) que en la evaluación inicial, el porcentaje que se sitúa en la población temporo-mandibular es inferior $(31.6 \%, n=12)$, y el porcentaje en la población psiquiátrica es ligeramente inferior (26.3\%, $n=10)$. La comparación entre la evaluación inicial y a los dos años, los podemos ver en la figura 2.

Respecto a la Escala de evaluación global que cubre el psicólogo de referencia, las puntuaciones medias en todas las áreas analizadas son inferiores a las obtenidas en la evaluación inicial (ver figura 3). A menor puntuación, mejor funcionamiento. Las puntuaciones a los dos años son las siguientes: en el área psicopatológica la puntuación media es de 0.94 (D.T. $=0.86)$, en el área familiar la puntuación media es de 1.02 (D.T. = 1.15), en el área laboral la puntuación media es de 0.71 (D.T. $=0.98)$, y en el área judicial la puntuación media es de 0.55 (D.T. = 1.13).

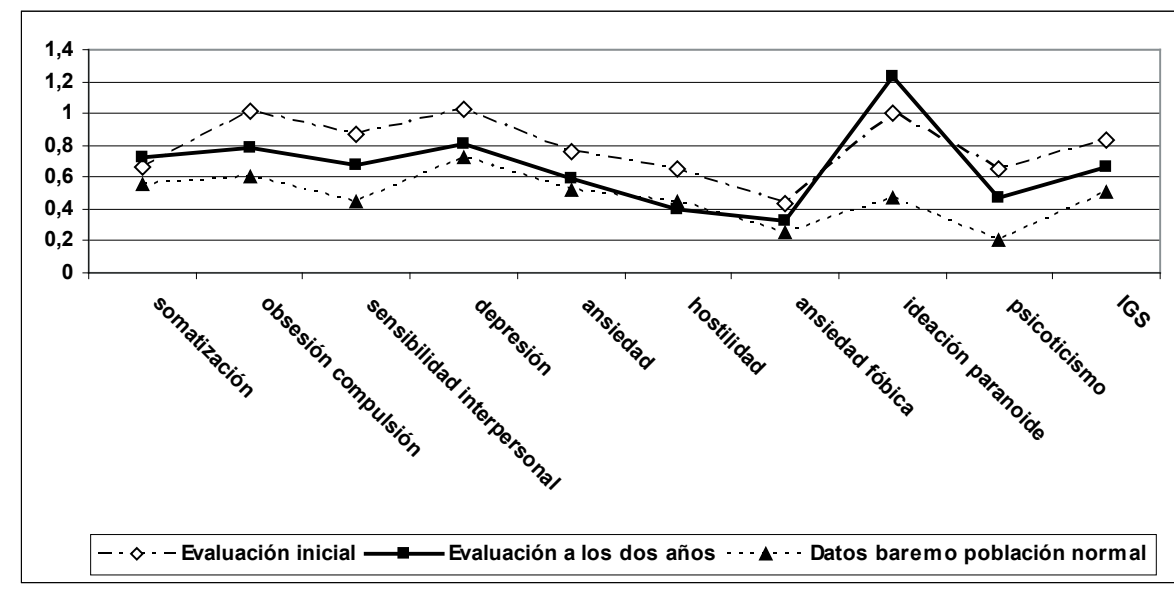

Figura 1. Puntuaciones medias en el SCL-90R: evaluación inicial, a los dos años y puntuaciones en población normal $(\mathbf{N}=\mathbf{3 8})$.

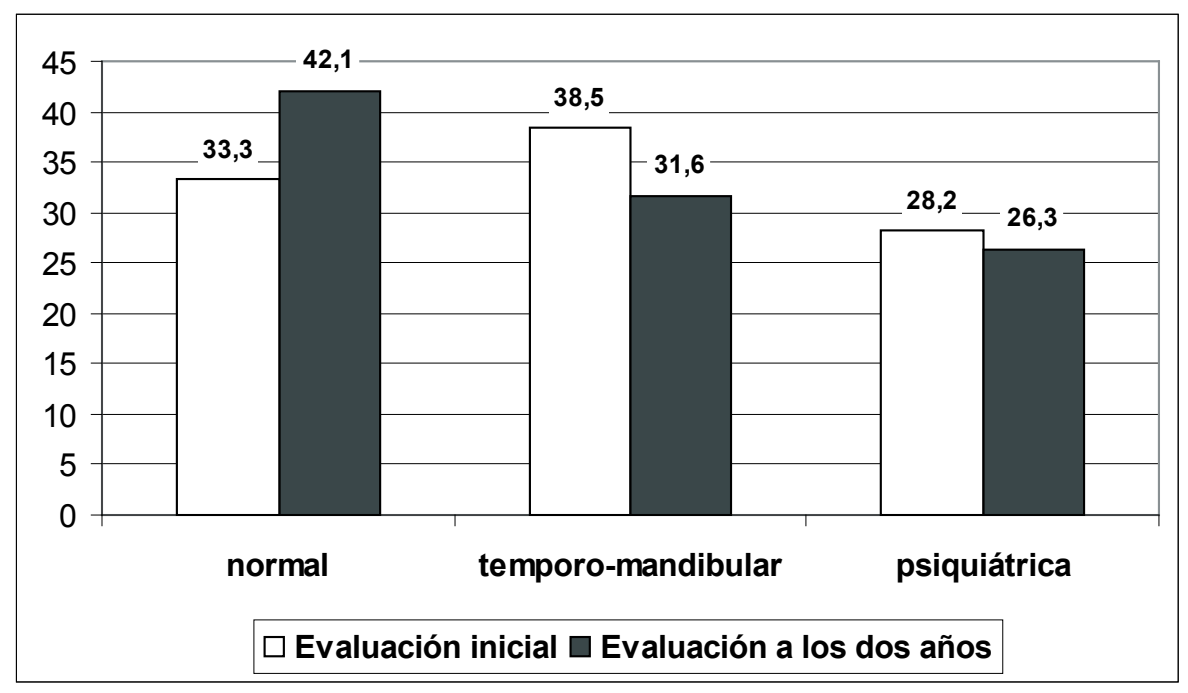

Figura 2. Clasificación de los sujetos en las tablas del SCL-90R: evaluación inicial y a los dos años ( $N=38)$. 


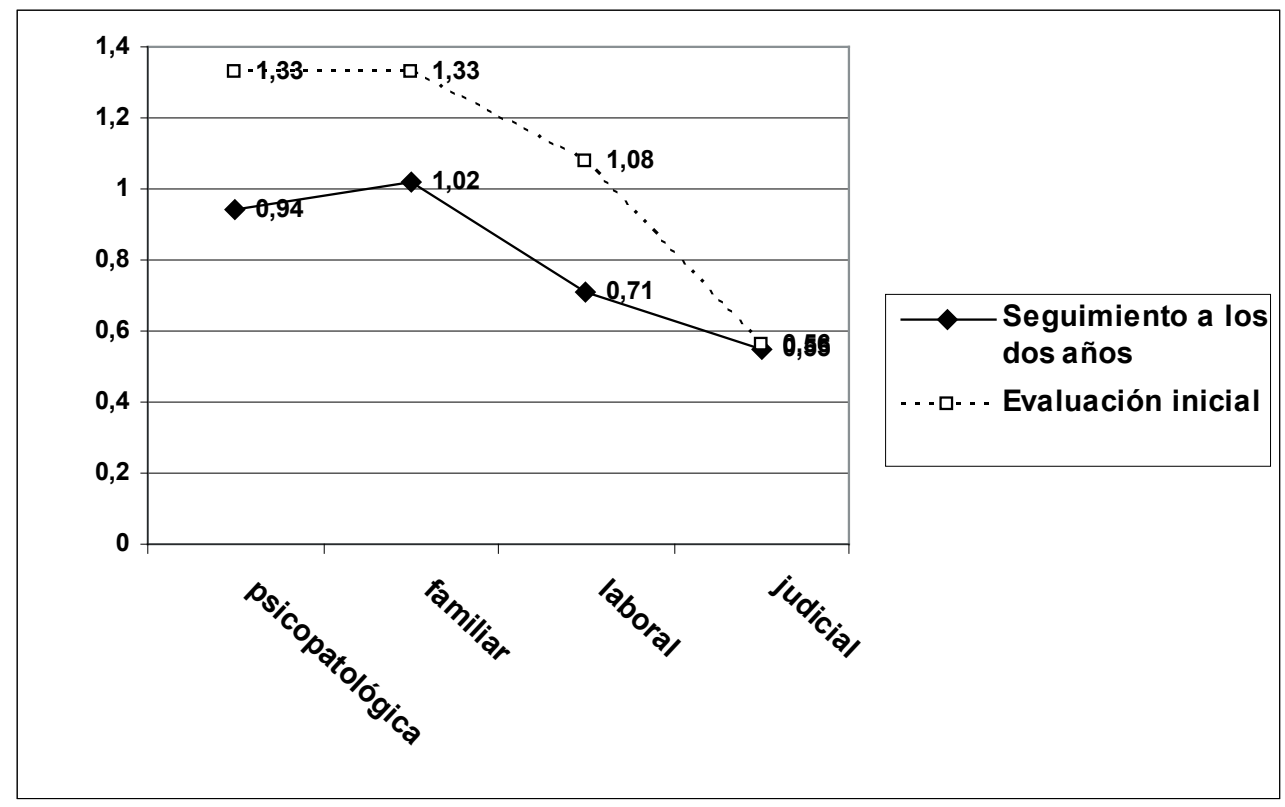

Figura 3. Escala de Evaluación Global que cubre el terapeuta de referencia: evaluación inicial y a los dos años $(\mathbf{N}=38)$.

\section{DISCUSIÓN}

Los resultados obtenidos en el presente estudio indican una evolución positiva, después de dos años, de una muestra de personas con dependencia de la cocaína que han recibido o reciben tratamiento en diversos Centros de Drogodependencias de la red pública de Galicia. En todas las áreas que han sido analizadas, en dicha muestra, los resultados obtenidos han sido muy positivos.

Aunque el tamaño de la muestra a los dos años es reducido $(N=38)$, en relación a la muestra inicial $(N=$ $115)$, el porcentaje de personas evaluadas 24 meses después de la evaluación inicial (el 33\% de la muestra inicial) es similar al de otros estudios de seguimiento de 12 meses (Carroll et al., 2000; Carroll et al., 1993).

Respecto al consumo de cocaína en el último mes, el porcentaje de personas que consumieron es muy bajo $(21.1 \%)$, si lo comparamos con otros estudios de seguimiento. En el estudio de seguimiento de 12 meses de Crist-Christoph et al. (1999), la media de días de consumo en los últimos 30 días es de 3.4 $(D . T .=6.5)$, mientras que en el presente estudio de seguimiento de dos años es de 1.18 (D.T. = 4.9). En el estudio de McKay, Merikle, Mulvaney, Weiss y Koppenhaver (2001), el 41\% de la muestra había consumido cocaína durante los seis meses previos a la evaluación de 24 meses.

Una de las dificultades principales que hemos encontrado para poder comparar los datos obteni- dos, es la escasez de estudios de seguimiento a largo plazo.

Analizando la evolución de estos sujetos partiendo de los dos estudios publicados previamente con esta misma muestra (López et al., 2006; López et al., 2007), observamos que los resultados obtenidos son muy satisfactorios: a los seis meses el porcentaje de sujetos abstinentes es del $30.4 \%$ del total de la muestra, y un $38 \%$ de los localizados. A los doce meses el $17.4 \%$ de la muestra total continúa abstinente en el consumo de cocaína desde la evaluación inicial (abstinencia continua), lo que representa un $21.5 \%$ de los sujetos localizados. Y a los 24 meses, utilizando un criterio de evaluación de la abstinencia menos estricto, los resultados son que el $26.0 \%$ de la muestra inicial no ha consumido cocaína en el último mes, un 78.9\% de los sujetos localizados. Este dato es relevante, ya que como señalábamos al principio es importante evaluar el consumo de sustancias en el mes previo, ya que predice la existencia de consumos en el futuro (Reiben et al., 2002).

Estudios de seguimiento más amplios, como el de 5 años realizado por Simpson, Joe y Broome (2002), apuntan que los resultados obtenidos al año de iniciar el tratamiento son similares a los que se obtienen 5 años después de haber iniciado el tratamiento. Por lo tanto, los resultados positivos que se obtienen al año se mantienen a lo largo del tiempo.

En cuanto al consumo de otras sustancias en el último mes, destaca el dato del consumo de tabaco: el $89.5 \%$ de la muestra ha fumado algún cigarrillo en 
el último mes. Es una prevalencia de consumo muy superior a la que encontramos en la población general, en donde el 38.4\% ha consumido en el último mes (PNSD, 2007). Por lo tanto, es muy relevante la alta prevalencia de consumo de tabaco en personas con dependencia de la cocaína. De modo semejante, el $73.7 \%$ de la muestra ha consumido alcohol en el último mes. Es un porcentaje algo superior al de la población general en la que el consumo en el último mes se sitúa en el 64.6\% (PNSD, 2007).

El consumo de cannabis también es más alto que en la población general: en el presente estudio el $36.8 \%$ de la muestra ha consumido cannabis en el último mes, mientras que en la población general el consumo en el último mes se sitúa en el 8.7\% (PNSD, 2007). El consumo de heroína en el último mes entre los sujetos del presente estudio (5.3\%) también es superior al de la población general (menos de un 1\% en el último mes; PNSD, 2007).

Por lo tanto, nos encontramos con una muestra de sujetos en los que el consumo de sustancias psicoactivas, diferentes a la cocaína que motiva el tratamiento, es muy superior al consumo de la población general.

Respecto a la evaluación realizada con el SCL-90-R, los datos indican una evolución positiva después de dos años de la evaluación inicial. Excepto en las subescalas de somatización e ideación paranoide, en el resto de las subescalas y en los índices generales hay una disminución de la puntuación media, y por lo tanto una disminución del malestar psicológico percibido por el paciente. Pero al igual que ocurre con el consumo de sustancias en el último mes, estas puntuaciones medias siguen siendo más elevadas que las obtenidas en la población general.

Respecto al ajuste social, algo más del 70\% de la muestra tiene un funcionamiento social normalizado, y el 73\% no tiene ningún problema judicial.

La evaluación que realiza el psicólogo de referencia confirma la evolución positiva de las personas que forman la muestra del presente estudio. En las áreas psicopatológica, familiar y laboral presentan un mejor funcionamiento a los dos años, y el área judicial presenta una puntuación similar.

De todo lo anterior, podemos hablar de una evolución positiva, tras dos años de seguimiento, de las personas con dependencia de la cocaína que demandaron tratamiento en Centros de Drogodependencias públicos de Galicia, que han sido evaluadas en el presente estudio. Esta evolución positiva se refleja tanto en las altas tasas de abstinencia en el consumo de cocaína en el último mes, como en el buen ajuste social, la disminución del malestar psicológico percibido y la escasa presencia de problemas judiciales.
Como principal limitación del presente estudio, debemos destacar el tamaño de la muestra analizada por no haber podido localizar a dos tercios de las personas que formaban la muestra inicial. En posteriores estudios debe de ser superior el tamaño de la muestra de la que se parte para poder hablar de una generalización de los resultados. Podemos considerar como otra limitación que sólo la mitad de la muestra ha realizado analíticas de orina para confirmar la abstinencia de cocaína. Sin embargo, estudios como el de Simpson, Joe y Broome (2002), apuntan la existencia de una alta coincidencia entre los resultados de las analíticas de orina y la información que da el paciente sobre la existencia o no de consumos de cocaína.

Consideramos que la importancia de este estudio radica en la inexistencia en nuestro país de estudios de este tipo que describan cómo es la evolución de las personas que están en tratamiento por problemas con el consumo de cocaína. Esto subraya la necesidad de continuar trabajando en esta línea para analizar el trabajo que se está llevando a cabo desde los Centros de Drogodependencias o de Conducta Adictivas.

\section{REFERENCIAS}

Beck, A. T., Rush, A. J., Shaw, B. F. y Emery, G. (1979). Cognitive Therapy of Depression. New York: Guildford Press (Trad. española en Bilbao: Desclée de Brower, 1983).

Bernstein, J., Bernstein, E., Tassiopoulos, K., Heeren, T., Levenson, S. y Hingson, R. (2005). Brief motivational intervention at a clinic visit reduces cocaine and heroine use. Drug and Alcohol Dependence, 77, 49-59.

Carroll, Nich, Ball, McCance, Frankforter y Rosaville (2000). One-year follow-up of disulfiram and psychotherapy for cocaine-alcohol users: sustained effects of treatment. Addiction, 95, 1335-1349.

Carroll, K. M., Power, M D., Bryant, K. y Rounsaville, B. J. (1993). One-year follow-up status of treatment-seeking cocaine abusers. Journal of Nervous and Mental Disease, 181, 71-79.

Crist-Christoph, P., Siqueland, L., Blaine, J., Frank, A., Luborsky, L., Onken, L. S., Muenz, L. R, Thase, M. E., Weiss, R. D., Gastfirnd, D. R., Woody, G. E., Barber, J. P., Butler, S. F., Daley, D., Salloum, I., Bishop, S., Najavits, L. M., Lis, J., Mercer, D., Griffin, M. L., Moras, K. y Beck, A. T. (1999). Psychosocial treatments for cocaine dependence. National Institute on Drug Abuse Colloborative Cocaine Treatment Study. Archives of General Psychiatry, 56, 493-501.

Derogatis, L. R. (2002). SCL-90-R. Cuestionario de 90 síntomas revisado. Manual. Madrid: Tea Ediciones.

First, M. B., Spitzer, R. L., Gibbson, M., Williams, J. B. W. y Smith-Benjamin, L. (1998). Guía del usuario de la 
entrevista clínica estructurada para los trastornos del eje I del DSM-IV- SCID-I. Barcelona: Masson.

González-Saiz, F.M., Salvador, L., Martínez, J.M., López, A., Ruz, I. y Guerra, D. (1997). Indicador del tratamiento de la adicción a opiáceos. Versión española de la entrevista clínica "Opiate Treatment Index": Manual. Cádiz: Universidad de Cádiz. Servicio de Publicaciones.

López, A., Becoña, E., García, J., Senra, A., Cancelo, J., Estévez, C., Sobradelo, J., Vieitez, I., Lloves, M., Moneo, A., Casete, L., Lage, M. y Díaz, E. (2006). ¿Cómo evolucionan las personas con dependencia de la cocaína que están en tratamiento? Estudio a los tres y seis meses. Adicciones, 18, 327-336.

López, A., Becoña, E., Lloves, M., Moneo, A., Vieitez, I., Cancelo, J., Estévez, C., Sobradelo, J., García, J., Senra, A., Casete, L., Lage, M. y Díaz, E. (2007). Evolución del tratamiento y consumo en personas con dependencia de la cocaína, a los doce meses, en función de la psicopatología asociada y el funcionamiento cotidiano. Adicciones, 19, 251-266.

McKay, J. R., Merikle, E., Mulvaney, F. D., Weiss, R. V. y Koppenhaver, J. M. (2001). Factors accounting for cocaine use two years following initiation of continuing care. Addiction, 96, 213-225.

McMahon, R. C., Kouzekanani, K. y Malow, R. M. (1999). A comparative study of cocaine-treatment completers and dropouts. Journal of Substance Abuse Treatment, $16,17-22$.

Millon, T. (1999). MCMI-II. Manual (adaptación española de Ávila-Espada, A., director; Jiménez, F., coordinador). Madrid: TEA.

Pedrero, E. J., Puerta, C., Segura, I. y Martínez, S. (2004). Evolución de la sintomatología psicopatológica de los drogodependientes a lo largo del tratamiento. Trastornos Adictivos, 6, 175-191.
Plan Nacional sobre Drogas (2007). Encuesta domiciliaria sobre alcohol y drogas en España 2005-2006 Análisis y principales resultados. Recuperado el 27 de septiembre de 2007 en http://www.pnsd.msc.es/ Categoria2/observa/pdf/Domiciliaria2005-2006.pdf

Reiber, CH., Ramírez, A., Parent, D. y Rawson, R. A. (2002). Predicting treatment success at multiple timepoints in diverse populations of cocaine-dependent individuals. Drug and Alcohol Dependence, 68, 35-48.

Sánchez-Hervás, E., Morales, E., Tomás, V., Molina, N., del Olmo, R., Bosch, C., Sanoguera, M. y Escrivá, P. (2002). Tratamiento de la dependencia a cocaína: estudio de seguimiento de seis meses. Adicciones, 14 (Supl.2), 137.

Saunders, J., Aasland, O., Babor, T., De la Fuente, J. y Grant, M. (1993). Development of the AUDIT: WHO collaborative project on early detection of persons with harmful alcohol consumption-II. Addiction, 88, 791-804.

Secades-Villa, R., García-Rodríguez, O., Álvarez, H., Río, A., Fernández-Hermida, J. L. y Carballo, J. L. (2007). El programa de reforzamiento comunitario más terapia de incentivo para el tratamiento de la adicción a la cocaína. Adicciones, 19, 51-57.

Simpson, D. D., Joe, G. W. y Broome, K. M. (2002). A national 5-year follow-up of treatments outcomes for cocaine dependence. Archives of General Psychiatry, $59,538-544$

Solé, J. (2001). Tratamiento del consumo de cocaína. Integrando psicoterapia y farmacoterapia. Adicciones, 13 (Sup.2), 209-225.

Spielberger, C. D., Gorsuch, R. L. y Luchene, R. E. (1971). Cuestionario de ansiedad estado-rasgo. Manual de la adaptación española, $4^{\text {a }}$ edición revisada. Madrid, TEA Ediciones. 


\section{Anexo 1. Escala de evaluación del funcionamiento global del paciente a cubrir por el terapeuta ( 24 meses)}

Indique, aproximadamente, la fecha de la última consulta que tuvo con este paciente

Indique si el paciente ha realizado nuevos consumos de cocaína desde que se realizó la evaluación psicológica:

SI

En el caso de que el paciente no haya acudido a las últimas citas, indíquelo con una $\mathrm{X}$ en el siguiente recuadro:

Señale con una $X$ en el recuadro, qué nivel de funcionamiento presenta el paciente en las cuatro siguientes áreas en el momento actual o en la última ocasión en que fue visto por el terapeuta:

\begin{tabular}{|c|c|c|c|c|c|}
\hline & 0 & 1 & 2 & 3 & 4 \\
\hline Psicopatología & $\begin{array}{l}\text { Ausencia de sín- } \\
\text { tomas }\end{array}$ & $\begin{array}{l}\text { Algunos síntomas } \\
\text { leves; ej. humor } \\
\text { depresivo o lige- } \\
\text { ras alteraciones } \\
\text { del sueño }\end{array}$ & $\begin{array}{l}\text { Síntomas psi- } \\
\text { copatológicos } \\
\text { moderados; ej. } \\
\text { distimia o crisis } \\
\text { de angustia oca- } \\
\text { sionales }\end{array}$ & $\begin{array}{l}\text { Trastorno psico- } \\
\text { patológico grave; } \\
\text { ej. trastorno de } \\
\text { depresión mayor } \\
\text { o crisis de angus- } \\
\text { tia frecuentes }\end{array}$ & $\begin{array}{l}\text { Alteración de la } \\
\text { realidad o de la } \\
\text { comunicación (ej. } \\
\text { lenguaje incohe- } \\
\text { rente o actúa de } \\
\text { forma inapropia- } \\
\text { da) o con peligro } \\
\text { de causar daño a } \\
\text { sí mismo o a los } \\
\text { demás }\end{array}$ \\
\hline $\begin{array}{l}\text { Ámbito familiar/ } \\
\text { social }\end{array}$ & $\begin{array}{l}\text { Funcionamiento } \\
\text { normalizado }\end{array}$ & $\begin{array}{l}\text { Algunas dificul- } \\
\text { tades (ej. discu- } \\
\text { siones con los } \\
\text { allegados), pero } \\
\text { en general funcio- } \\
\text { na bastante bien } \\
\text { y tiene relaciones } \\
\text { interpersonales } \\
\text { significativas }\end{array}$ & $\begin{array}{l}\text { Dificultades } \\
\text { moderadas } \\
\text { (ej. pequeños } \\
\text { robos en casa o } \\
\text { frecuentes discu- } \\
\text { siones) }\end{array}$ & $\begin{array}{l}\text { Dificultades } \\
\text { importantes en } \\
\text { las relaciones } \\
\text { (ej. dificultades } \\
\text { graves de convi- } \\
\text { vencia) }\end{array}$ & $\begin{array}{l}\text { Alteración grave } \\
\text { en el área de las } \\
\text { relaciones (ej. } \\
\text { abandono de la } \\
\text { familia) }\end{array}$ \\
\hline $\begin{array}{l}\text { Ámbito laboral/ } \\
\text { académico }\end{array}$ & $\begin{array}{l}\text { Funcionamiento } \\
\text { normalizado }\end{array}$ & $\begin{array}{l}\text { Algunas dificul- } \\
\text { tades (ej. faltas } \\
\text { ocasionales al } \\
\text { trabajo o a la } \\
\text { escuela) pero en } \\
\text { general funciona } \\
\text { bastante bien }\end{array}$ & $\begin{array}{l}\text { Dificultades } \\
\text { moderadas (ej. } \\
\text { conflictos con los } \\
\text { compañeros de } \\
\text { trabajo o estu- } \\
\text { dios) }\end{array}$ & $\begin{array}{l}\text { Dificultades } \\
\text { importantes (ej. } \\
\text { incapacidad de } \\
\text { mantener un } \\
\text { empleo) }\end{array}$ & $\begin{array}{l}\text { Alteración grave } \\
\text { en el área laboral/ } \\
\text { académica (ej. } \\
\text { incapaz de traba- } \\
\text { jar o estudiar, o } \\
\text { permanece en la } \\
\text { cama todo el día) }\end{array}$ \\
\hline Ámbito judicial & $\begin{array}{l}\text { No tiene proble- } \\
\text { mas legales }\end{array}$ & $\begin{array}{l}\text { Acusado de } \\
\text { cometer delitos } \\
\text { leves (ej. tenen- } \\
\text { cia de drogas o } \\
\text { estar implicado } \\
\text { en altercados) }\end{array}$ & $\begin{array}{l}\text { Acusado de } \\
\text { cometer delitos } \\
\text { importantes (ej. } \\
\text { tráfico de drogas } \\
\text { o robo) }\end{array}$ & $\begin{array}{l}\text { Se encuentra en } \\
\text { libertad condi- } \\
\text { cional }\end{array}$ & $\begin{array}{l}\text { Se encuentra } \\
\text { cumpliendo una } \\
\text { condena en la } \\
\text { actualidad }\end{array}$ \\
\hline
\end{tabular}

* El objetivo es evaluar, desde la perspectiva del terapeuta, el nivel de deterioro del sujeto en distintas áreas para poder determinar cuáles son las que precisan una intervención más inmediata y facilitar la realización de una evaluación a lo largo del tiempo de los cambios que se van produciendo con el tratamiento 
